\title{
Heart rate variability biofeedback in patients with alcohol dependence: a randomized controlled study
}

This article was published in the following Dove Press journal:

Neuropsychiatric Disease and Treatment

9 October 2015

Number of times this article has been viewed

\author{
Ana Isabel Penzlin' \\ Timo Siepmann² \\ Ben Min-Woo Illigens ${ }^{3}$ \\ Kerstin Weidner ${ }^{4}$ \\ Martin Siepmann ${ }^{4}$ \\ Institute of Clinical Pharmacology, \\ 2Department of Neurology, \\ University Hospital Carl Gustav \\ Carus, Technische Universität \\ Dresden, Dresden, Saxony, Germany; \\ ${ }^{3}$ Department of Neurology, Beth \\ Israel Deaconess Medical Center, \\ Harvard Medical School, Boston, MA \\ USA; ${ }^{4}$ Department of Psychotherapy \\ and Psychosomatic Medicine, \\ University Hospital Carl Gustav \\ Carus, Technische Universität \\ Dresden, Dresden, Saxony, Germany
}

Correspondence: Martin Siepmann Department of Psychotherapy and Psychosomatic Medicine, University Hospital Carl Gustav Carus, Technische Universität Dresden, Fetscherstraße 74, $0 \mid 307$ Dresden

Tel +4935 I 458 I 7094

Fax +49 35I 4586332

Email martin.siepmann@mailbox. tu-dresden.de
Background and objective: In patients with alcohol dependence, ethyl-toxic damage of vasomotor and cardiac autonomic nerve fibers leads to autonomic imbalance with neurovascular and cardiac dysfunction, the latter resulting in reduced heart rate variability (HRV). Autonomic imbalance is linked to increased craving and cardiovascular mortality. In this study, we sought to assess the effects of HRV biofeedback training on HRV, vasomotor function, craving, and anxiety.

Methods: We conducted a randomized controlled study in 48 patients ( 14 females, ages $25-59$ years) undergoing inpatient rehabilitation treatment. In the treatment group, patients $(n=24)$ attended six sessions of HRV biofeedback over 2 weeks in addition to standard rehabilitative care, whereas, in the control group, subjects received standard care only. Psychometric testing for craving (Obsessive Compulsive Drinking Scale), anxiety (Symptom Checklist-90-Revised), HRV assessment using coefficient of variation of R-R intervals (CVNN) analysis, and vasomotor function assessment using laser Doppler flowmetry were performed at baseline, immediately after completion of treatment or control period, and 3 and 6 weeks afterward (follow-ups 1 and 2).

Results: Psychometric testing showed decreased craving in the biofeedback group immediately postintervention (OCDS scores: $8.6 \pm 7.9$ post-biofeedback versus $13.7 \pm 11.0$ baseline [mean \pm standard deviation], $P<0.05$ ), whereas craving was unchanged at this time point in the control group. Anxiety was reduced at follow-ups 1 and 2 post-biofeedback, but was unchanged in the control group $(P<0.05)$. Following biofeedback, CVNN tended to be increased $(10.3 \% \pm 2.8 \%$ post-biofeedback, $10.1 \% \pm 3.5 \%$ follow-up $1,10.1 \% \pm 2.9 \%$ follow-up 2 versus $9.7 \% \pm 3.6 \%$ baseline; $P=$ not significant). There was no such trend in the control group. Vasomotor function assessed using the mean duration to $50 \%$ vasoconstriction of cutaneous vessels after deep inspiration was improved following biofeedback immediately postintervention and was unchanged in the control group $(P<0.05)$.

Conclusion: Our data indicate that HRV biofeedback might be useful to decrease anxiety, increase HRV, and improve vasomotor function in patients with alcohol dependence when complementing standard rehabilitative inpatient care.

Keywords: HRV, CVNN, alcohol addiction, rehabilitation, craving, laser Doppler flowmetry

\section{Introduction}

Although multimodal alcohol rehabilitation programs are effective and widely used, chronic alcohol addiction is still a global medical and socioeconomic problem, with 14.6 million affected patients in Europe alone, and is linked to increased cardiovascular mortality mediated by cardiac and vascular autonomic dysfunction. ${ }^{1,2}$ In patients with chronic alcohol addiction, ethyl-toxic damage of vasomotor and cardiac autonomic 
nerve fibers leads to neurovascular dysfunction and decreased heart rate variability (HRV). ${ }^{3}$ While HRV constitutes a composite measure of balance of sympathetic and parasympathetic tones, in alcoholic neuropathy, functional and structural damage of both sympathetic and parasympathetic nerve fibers individually contribute to impaired autonomic cardiac function. ${ }^{4,5}$ This cardiac autonomic disbalance is linked to increased alcohol craving. ${ }^{6}$ In turn, elevated craving is related to higher rates of relapse after addiction treatment. ${ }^{7-9}$ To date, there are no interventions to specifically treat autonomic dysfunction and consequentially decrease craving.

In autonomic dysfunction related to other psychiatric diseases such as depression and anxiety, biofeedback targeting HRV via visualization of heart rate deviations on a computer screen was demonstrated to improve cardiac autonomic function by increasing HRV and to alleviate depressive symptoms as well as symptoms of anxiety. ${ }^{10,11}$ A recent pilot study demonstrated that HRV biofeedback in a short-term setting (three single biofeedback sessions) is feasible to treat patients with substance use disorder, but found no positive effect of the intervention on craving symptoms or HRV. ${ }^{12}$ However, this study was limited by the short duration of the intervention and a heterogeneous study population which also included patients addicted to drugs. Therefore, it remains to be answered whether these negative results were due to overestimation of efficacy or heterogeneity, respectively.

In this study, we aimed to assess the hypothesis that HRV biofeedback increases HRV, improves peripheral autonomic function, and decreases craving in patients with alcohol dependence and might therefore supplement other treatment regimens of chronic alcohol addiction.

\section{Methods}

\section{Subjects and protocol}

Male and female patients undergoing inpatient rehabilitation treatment for alcohol use disorder were considered for participation in the study if they were older than 18 years of age, met the Diagnostic and Statistical Manual of Mental Disorders, 4th Edition criteria for alcohol dependence, and had achieved at least 5 days of abstinence prior to study entrance. The presence of withdrawal syndrome was excluded by clinical interview and physical examination, as absence of withdrawal symptoms is also a prerequisite of rehabilitative treatment following our institutional standard. Eligible patients were enrolled by the conducting study physician. With the use of a computer-generated random-allocation sequence, participants were assigned by the conducting study physician in a simple randomization fashion in a $1: 1$ ratio to receive either HRV biofeedback in addition to standard rehabilitative care or standard rehabilitative care only. To avoid confounding by comorbidities influencing autonomic functions or psychometric assessment, we excluded subjects with diabetes mellitus, any heart diseases, depression, schizophrenia, bipolar disorder, or dementia. Additionally, we excluded those patients that were treated with any antidepressant, beta blocker, alpha blocker, or cholinergic or anticholinergic drug within 3 months prior to the study to avoid pharmacodynamic confounding. Patients in the HRV biofeedback group underwent three sessions of HRV biofeedback training per week over 2 weeks, whereas control patients did not undergo biofeedback. Psychometric questioning and assessment of autonomic functions were performed before the beginning of the first biofeedback session (baseline), immediately after completion of the last biofeedback session, and 3 and 6 weeks after the last biofeedback session (follow-ups 1 and 2). Study assessments in the control group were performed with the same time intervals. Adherence was further facilitated by reminders to attend study procedures by our nurses, physicians, and therapists.

The application of the study intervention was performed in an open setting where neither the patient nor the investigator was blinded to the group allocation (control or biofeedback). The investigator who analyzed the results of psychometric and autonomic assessment as well as the statistician who performed statistical analysis of the data were blinded to group allocation.

\section{Ethical standards}

Written informed consent was obtained from each participant prior to the study. The study was approved by the local institutional review board (Ethikkommission an der Technischen Universität Dresden; IRB number: EK118042010). The study was registered in the German Clinical Trials Register (DRKS00004618).

\section{Standard rehabilitative care}

All study patients received standard inpatient care at the Heidehof Hospital rehabilitative treatment center for substance use disorder, Weinböhla, Saxony, Germany. Standard care comprised daily 90-minute sessions of cognitive behavioral group psychotherapy, individual 1-hour sessions of motivational interviewing and cognitive behavioral therapy once a week, 1-hour psychoeducational group sessions three times per week, and daily 3-hour sessions of occupational therapy. 


\section{HRV biofeedback}

Biofeedback is a behavioral intervention that enables patients to achieve voluntary control over physiological processes due to changes in behavior. Electronic measurements of a physiological function (eg, HRV) are coupled with a feedback signal that changes whenever patients succeed or fail to achieve the desired changes in bodily processes due to their behavioral modifications (eg, breathing). In our study, a validated HRV biofeedback system (StressPilot ${ }^{\mathrm{TM}}$; BioSign, Ottenhofen, Germany) was used as described previously. ${ }^{11}$ In brief, patients would sit in a comfortable chair in front of a computer monitor. The pulse wave was continuously measured in the earlobe, and HRV was calculated. Real-time visualization of HRV was achieved by a digital balloon on the computer screen which moved up and down proportionally reflecting the amplitude of HRV. With an increase or decrease in HRV the balloon was visualized as either rising or falling on the screen. At the beginning of the HRV biofeedback training, patients were orally instructed by the study physician to breathe at a given frequency of six cycles per minute following the given pacing stimulus, as this frequency was previously shown to yield maximum amplitudes of HRV. ${ }^{13,14}$ As breath-pacing stimulus, we used a bar on the computer screen that moved up and down to indicate whether patients should breathe in or out. Thus, paced breathing at six cycles per minute to increase HRV in a visual biofeedback setting was the intervention in this study. HRV biofeedback was conducted for 20 minutes at each session. Patients in the HRV biofeedback group underwent three sessions of HRV biofeedback training per week over 2 weeks, with 2 or 3 days of interval between the sessions.

\section{Psychometric testing}

Psychiatric diagnoses were assessed according to DSM-IV by a board-certified psychiatrist and documented using the structured clinical interview for DSM-IV. Perceived craving for alcohol was assessed using the Obsessive Compulsive Drinking Scale (OCDS). ${ }^{15}$ This 14 -item, self-administered instrument assesses efforts and abilities to resist thoughts of alcohol and drinking as well as impulses to drink. The questions use descriptors anchored on numerical ratings ranging from 0 to 4 , where higher scores indicate higher craving intensities. Because of the nondrinking prerequisite of our institutional inpatient treatment protocol, items 7 and 8 (questions on the amount of current drinking) were excluded. ${ }^{16}$

Psychopathology was assessed using the Symptom Checklist-90-Revised (SCL-90-R), a self-report instrument composed of a 90 -item questionnaire assessing the subscales anxiety, depression, interpersonal sensitivity, somatization, obsessive-compulsive, phobic-anxiety, hostility, psychoticism, and paranoid ideation. ${ }^{17}$ Items are quantified by a numeric rating scale from 0 to 4 (0 meaning symptom not present, 4 meaning very high symptom intensity). Depressive symptoms were also assessed using the Beck Depression Inventory-II (BDI-II). The BDI-II is a 21-item, self-administered questionnaire that measures affective, cognitive, and autonomic characteristics of depression. ${ }^{18}$ Each item is rated from 0 to 3 , reflecting symptom severity.

\section{Cardiac autonomic function assessment: HRV}

Analysis of HRV was carried out using a physiological signal analysis software package (Chart $5^{\circledR}$; AD Instruments, Castle Hill, Australia) as previously described. ${ }^{19}$ Briefly, the electrocardiogram signal was digitized using a sample rate of 400 per second. HRV was assessed following a resting period of 10 minutes. Respiration was monitored by registration of chest movements using a thoracic belt respiration transducer (Pneumotrace ${ }^{\circledR}$ II 1132; UFI, Morro Bay, CA, USA). The coefficient of variation of R-R intervals (CVNN) was calculated from 200 heartbeats following manual artefact removal, and spectral power analysis was carried out by means of a fast Fourier transformation. Absolute power values were assessed for two frequency bands: low frequency (LF), 0.04-0.15 Hz; and high frequency (HF), to 0.15-0.4 $\mathrm{Hz}$, as well as for total power (TP).

To determine the HRV under paced breathing, subjects were instructed to breathe deeply at a frequency of six cycles per minute. This pattern of paced breathing was achieved by asking the participants to synchronize their breathing with breathe-in and breathe-out guiding tones given via headphones. The HRV parameters CVNN, LF, HF, and TP were calculated in the same fashion as in the abovementioned resting condition analyses.

\section{Vasomotor autonomic function: laser Doppler flowmetry}

Laser Doppler flowmetry (LDF) was used to quantify vasoconstrictory responses of cutaneous blood vessels induced by sympathetic stimulation. The cutaneous blood flow was measured in relative units using a laser Doppler flow meter (Periflux ${ }^{\circledR}$; Perimed, Järfälla, Sweden) as previously described. ${ }^{20}$ Briefly, the laser Doppler probe was affixed to the palmar aspect of the distal phalanx of the index to quantify arterial, capillary, and venous erythrocyte flow at a depth of $2 \mathrm{~mm}$. After a resting period of 10 minutes, patients were 
instructed to take a single deep breath. The decrease and consecutive increase of cutaneous flow provoked by deep respiration (also referred to as inspiratory gasp response) was evaluated to assess sympathetic vasomotor function with temporal resolution: durations to $50 \%$ constriction $(\Delta \mathrm{t} 50 \%$ down) and $50 \%$ redilation of cutaneous vessels $(\Delta \mathrm{t} 50 \%$ up) were calculated.

\section{Statistics}

Statistical analyses were performed using the Sigma Stat ${ }^{\circledR}$ software package (Jandel, San Rafael, CA, USA). A power calculation was performed prior to the study. Based on the results of a previous study observing a standard deviation of 2.2 in CVNN, the primary outcome variable in our study, the sample size of $n=48$ was estimated, to result in a difference of $19.2 \%$ between HRV biofeedback and control with a power of $0.8 .{ }^{21}$ Outcome data are expressed as means and standard deviations. Psychometric measures and measures of autonomic function were compared between patients undergoing HRV biofeedback and control patients using a one-way analysis of variance procedure for repeated measurements following testing for normality and equality of variances. Statistical significance was accepted at $P<0.05$. Wherever significant, Bonferroni post hoc tests were used for comparisons between groups. $t$-tests were used to test for baseline intergroup differences in age, sex, size, weight, tobacco use, and most frequent alcohol-related comorbidities. A multivariate sequential regression model was built to impute missing data.

\section{Results}

\section{Demographic and baseline parameters}

We included 48 patients with alcohol dependence (34 males and 14 females; ages 25-59 years; 42 \pm 7.8 , mean \pm standard deviation). Between the biofeedback group and the control group, there were no differences in baseline characteristics including age, sex, weight, size, tobacco use, and alcoholrelated comorbidities (Table 1). We documented three screening failures: two patients fulfilled the exclusion criterion depression, and one patient decided to leave our clinic immediately after the screening procedure.

\section{Missing data and adherence}

The total fraction of missing data in our dataset was $5.4 \%$. All missing data were missing completely at random. These missing data were distributed among time points of assessment as follows: $31.6 \%$ at baseline, $21 \%$ immediately after completion of HRV biofeedback or control interval, $31.6 \%$ at follow-up 1 , and $15.8 \%$ at follow-up 2. In HRV assess
Table I Demographic characteristics

\begin{tabular}{|c|c|c|c|}
\hline & $\begin{array}{l}\text { HRV biofeedback } \\
(n=24)\end{array}$ & $\begin{array}{l}\text { Control } \\
(n=24)\end{array}$ & $P$-value \\
\hline Age (years) & $40( \pm 7)$ & $44( \pm 8)$ & 0.06 \\
\hline Weight (kg) & $72( \pm I I)$ & $74( \pm 19)$ & 0.3 \\
\hline Size $(\mathrm{cm})$ & $174( \pm 9)$ & $174( \pm 10)$ & 0.49 \\
\hline Sex (\%) & $70.8 \mathrm{~m}, 29.2 \mathrm{f}$ & $70.8 \mathrm{~m}, 29.2 \mathrm{f}$ & 0.5 \\
\hline Tobacco use (\%) & 79 & 75 & 0.36 \\
\hline \multicolumn{4}{|l|}{ Comorbidities } \\
\hline Neuropathy (\%) & 33.3 & 16.7 & 0.09 \\
\hline Hepatic steatosis (\%) & 20.8 & 37.5 & 0.09 \\
\hline Liver cirrhosis (\%) & 4.2 & 12.5 & 0.07 \\
\hline
\end{tabular}

Notes: There were no differences in size, weight, sex, tobacco use, and comorbidities between groups. Data are expressed as either \% or mean \pm standard deviation. Abbreviations: $\mathrm{f}$, female; HRV, heart rate variability; m, male.

(CVNN, LF, HF, TP), we noted a total of $13.5 \%$ missing data in the intervention group and $6.5 \%$ in the control group. Missing data in the HRV biofeedback group occurred in seven patients $(29.2 \%)$. In the control group, missing data occurred in four patients (16.7\%). In vasomotor assessment $(\Delta \mathrm{t} 50 \%$ down, $\Delta \mathrm{t} 50 \%$ up) and psychometric testing (OCDS, SCL-90-R, SCL-90-R anxiety, SCL-90-R depression, BDI-II), no missing data were noted. All missing data were due to technical recording errors. In our study, there were no dropouts and no patients lost to follow-ups. Adherence to the study protocol was achieved by integrating the study protocol into the rehabilitative inpatient treatment protocol of our clinic. Since patients are treated in our clinic over 12 weeks, we were able to perform all follow-up measurements during the inpatient period of treatment.

\section{Craving}

We observed a decrease in craving measured by the OCDS in the HRV biofeedback group immediately after completion of the intervention and at follow-ups 1 and 2 compared to baseline (Table 2). In the control group, OCDS score was also decreased, but this reduction occurred after a delay, namely, at follow-ups 1 and 2.

\section{Anxiety and depression}

In the HRV biofeedback group, anxiety assessed using the SCL-90-R anxiety items was decreased at follow-ups 1 and 2 when compared to baseline, but there was no such change in the control group (Table 2). Opposing the observed reduction of anxiety after HRV biofeedback, the total SCL-90-R score (a composite measure of psychopathology) as well as depression assessed using BDI-II and SCL-90-R depression items were unchanged in both groups at each time point of evaluation (Table 2). 
Table 2 Psychometric evaluation

\begin{tabular}{lllllll}
\hline Intervention & Time & Scale & & & \\
\cline { 2 - 6 } & & $\begin{array}{l}\text { OCDS } \\
\text { craving }\end{array}$ & $\begin{array}{l}\text { SCL-90-R } \\
\text { total }\end{array}$ & $\begin{array}{l}\text { SCL-90-R } \\
\text { anxiety }\end{array}$ & $\begin{array}{l}\text { SCL-90-R } \\
\text { depression }\end{array}$ & $\begin{array}{l}\text { BDI-II } \\
\text { depression }\end{array}$ \\
\hline HRV biofeedback & Baseline & $13.7( \pm I I .0)$ & $46.6( \pm 33.5)$ & $5.8( \pm 4.5)$ & $9.7( \pm 7.2)$ & $7.6( \pm 6.0)$ \\
$\mathrm{n}=24$ & Postintervention & $8.6( \pm 7.9)^{*}$ & $38.0( \pm 37.0)$ & $3.7( \pm 5.1)$ & $7.4( \pm 7.9)$ & $7.0( \pm 9.1)$ \\
& Follow-up I & $7.5( \pm 6.9)^{*}$ & $30.3( \pm 25.4)$ & $2.9( \pm 3.0)^{*}$ & $5.9( \pm 6.0)$ & $5.8( \pm 9.1)$ \\
& Follow-up 2 & $7.6( \pm 6.7)^{*}$ & $31.4( \pm 21.9)$ & $2.7( \pm 2.9)^{*}$ & $5.8( \pm 4.8)$ & $5.3( \pm 8.2)$ \\
Control & Baseline & $11.6( \pm 7.4)$ & $45.3( \pm 45.8)$ & $3.6( \pm 3.7)$ & $10.0( \pm 7.4)$ & $9.0( \pm 7.3)$ \\
$\mathrm{n}=24$ & Postintervention & $7.2( \pm 7.8)$ & $37.0( \pm 28.9)$ & $3.1( \pm 2.9)$ & $8.6( \pm 7.3)$ & $7.2( \pm 6.6)$ \\
& Follow-up I & $3.6( \pm 3.6)^{*}$ & $37.8( \pm 36.9)$ & $2.8( \pm 2.7)$ & $7.5( \pm 8.2)$ & $6.5( \pm 8.8)$ \\
& Follow-up 2 & $3.2( \pm 3.6)^{*}$ & $33.0( \pm 31.5)$ & $2.2( \pm 1.9)$ & $6.8( \pm 8.1)$ & $5.7( \pm 7.6)$ \\
\hline
\end{tabular}

Notes: Decreases in craving were noted in the HRV biofeedback group and with a delay in the control group, whereas anxiety was decreased only in the biofeedback group. Data are expressed mean \pm standard deviation. $* P<0.05$ versus baseline. In the control group, the term "postintervention" refers to the post-control period.

Abbreviations: BDI-II, Beck Depression Inventory-II; HRV, heart rate variability; OCDS, Obsessive Compulsive Drinking Scale; SCL-90-R, Symptom Checklist-90-Revised.

\section{Cardiac autonomic function: HRV}

We noted a nonsignificant trend toward increase in CVNN following HRV biofeedback training compared to baseline and control, both under resting conditions (Figure 1) and under paced breathing (Figure 2). The spectral analysis parameters LF, HF, and TP assessed under resting conditions were unchanged after HRV biofeedback training compared to baseline and control (Table 3). This was also true for paced breathing (Table 4).

\section{Vasomotor autonomic function}

Vasomotor function assessed using $\Delta \mathrm{t} 50 \%$ down after deep inspiration was increased in the biofeedback group immediately after biofeedback training compared to baseline, but there was no such change at follow-ups 1 and 2 (Figure 3).

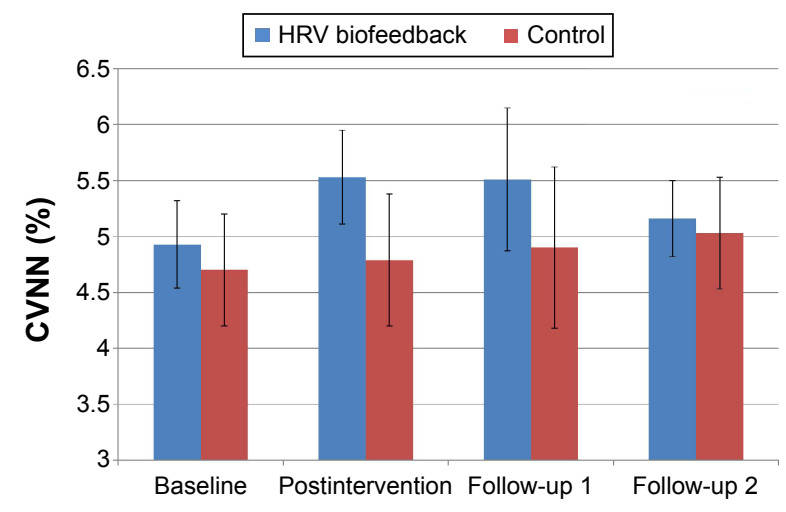

Figure I CVNN under resting conditions.

Notes: The bar graph illustrates a trend toward increased CVNN in HRV biofeedbacktreated patients, indicating possible improved function of cardiac autonomic nerve fibers. Data are presented as mean \pm standard deviation. $P=n$ s compared to baseline and control. HRV biofeedback: $n=24$; control: $n=24$. In the control group, the term "postintervention" refers to the post-control period.

Abbreviations: CVNN, coefficient of variation of R-R intervals; HRV, heart rate variability; ns, not significant.
In the control group, there was no such change. $\Delta \mathrm{t} 50 \%$ up was unchanged in the biofeedback group at each time point of evaluation compared with baseline and also remained unchanged in the control group (Figure 4).

\section{Discussion}

The major findings of our study are that patients with alcohol dependence in rehabilitative treatment receiving HRV biofeedback 1) perceived reduction of craving sooner than patients receiving only rehabilitative care but no HRV biofeedback; 2) showed a decrease in anxiety following the intervention; 3) tended to show improved cardiac autonomic function, reflected by a trend toward increased HRV postbiofeedback; and 4) showed improved vasomotor function after completion of training.

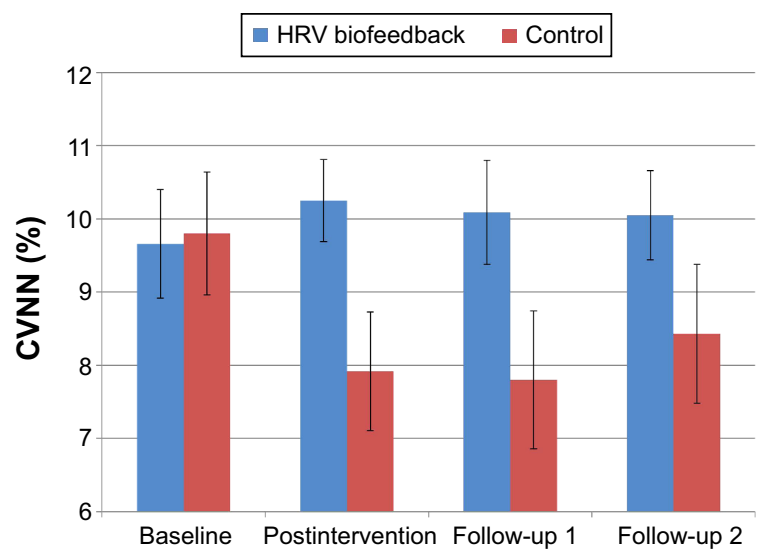

Figure $2 \mathrm{CVNN}$ under paced breathing conditions.

Notes: The bar graph illustrates a trend toward increased CVNN in HRV biofeedbacktreated patients, indicating possible improved function of cardiac autonomic nerve fibers. Data are presented as mean \pm standard deviation. $P=n s$ compared to baseline and control. HRV biofeedback: $n=24$; control: $n=24$. In the control group, the term "postintervention" refers to the post-control period.

Abbreviations: CVNN, coefficient of variation of R-R intervals; HRV, heart rate variability. 
Table 3 HRV parameters under resting conditions: spectral analysis

\begin{tabular}{|c|c|c|}
\hline \multirow[t]{2}{*}{ Parameter } & \multicolumn{2}{|l|}{ Resting condition } \\
\hline & $\begin{array}{l}\text { HRV biofeedback } \\
n=24\end{array}$ & $\begin{array}{l}\text { Control } \\
n=24\end{array}$ \\
\hline \multicolumn{3}{|l|}{ Baseline } \\
\hline $\mathrm{HF}\left(\mathrm{ms}^{2}\right)$ & $809.5( \pm I, 565.0)$ & $438.6( \pm 606.6)$ \\
\hline $\operatorname{LF}\left(\mathrm{ms}^{2}\right)$ & $656.0( \pm 858.0)$ & $463.1( \pm 462.4)$ \\
\hline $\mathrm{TP}\left(\mathrm{ms}^{2}\right)$ & I,90।.6 $( \pm 2,096.9)$ & $2,395.6( \pm 3,9 \mid 5.7)$ \\
\hline \multicolumn{3}{|c|}{ Postintervention } \\
\hline $\mathrm{HF}\left(\mathrm{ms}^{2}\right)$ & $762.3( \pm 1,198.9)$ & $662.8( \pm I, 426.1)$ \\
\hline $\operatorname{LF}\left(\mathrm{ms}^{2}\right)$ & $673.7( \pm 597.0)$ & $659.5( \pm 1,266.3)$ \\
\hline $\mathrm{TP}\left(\mathrm{ms}^{2}\right)$ & $1,946.0( \pm I, 933.3)$ & $1,839.5( \pm 3,181.2)$ \\
\hline \multicolumn{3}{|l|}{ Follow-up I } \\
\hline $\mathrm{HF}\left(\mathrm{ms}^{2}\right)$ & $420.2( \pm 43 \mid .3)$ & $568.6( \pm I, 23 \mid .2)$ \\
\hline $\operatorname{LF}\left(m s^{2}\right)$ & $695.3( \pm 748.0)$ & $\mathrm{I}, 123.7( \pm 3,681.3)$ \\
\hline $\mathrm{TP}\left(\mathrm{ms}^{2}\right)$ & $2,|I| 0.8( \pm 2,33 \mid .3)$ & $2,382.4( \pm 5,068.1)$ \\
\hline \multicolumn{3}{|l|}{ Follow-up 2} \\
\hline $\mathrm{HF}\left(\mathrm{ms}^{2}\right)$ & $358.7( \pm 304.7)$ & $428.2( \pm 6 \mid 5.5)$ \\
\hline $\operatorname{LF}\left(\mathrm{ms}^{2}\right)$ & $675.0( \pm 436.8)$ & $546.0( \pm 7 \mid 5.0)$ \\
\hline $\mathrm{TP}\left(\mathrm{ms}^{2}\right)$ & $1,638.3( \pm 926.4)$ & $\mathrm{I}, 768.4( \pm 2,029.2)$ \\
\hline
\end{tabular}

Notes: HRV was unchanged in both the control group and the biofeedback group. In the control group, the term "postintervention" refers to the post-control period. Data are expressed as mean \pm standard deviation. $P=n$ s.

Abbreviations: HF, high frequency; HRV, heart rate variability; LF, low frequency; TP, total power; ns, not significant.

The mechanism whereby HRV biofeedback improves autonomic functions is not fully elucidated. It was hypothesized that the intervention increases balance between the sympathetic and parasympathetic branches, but the contribution of each branch remains speculative. ${ }^{12}$ In psychiatric diseases related to a shift toward increased sympathetic and decreased parasympathetic tone such as anxiety and depression, HRV biofeedback was shown to improve both

Table 4 HRV parameters under paced breathing: spectral analysis

\begin{tabular}{|c|c|c|}
\hline \multirow[t]{2}{*}{ Parameter } & \multicolumn{2}{|l|}{ Paced breathing } \\
\hline & $\begin{array}{l}\text { HRV biofeedback } \\
n=24\end{array}$ & $\begin{array}{l}\text { Control } \\
n=24\end{array}$ \\
\hline \multicolumn{3}{|l|}{ Baseline } \\
\hline $\mathrm{HF}\left(\mathrm{ms}^{2}\right)$ & $\mathrm{I}, 565.4( \pm \mathrm{I}, 440.9)$ & $898.8( \pm I, 160.3)$ \\
\hline $\operatorname{LF}\left(\mathrm{ms}^{2}\right)$ & $4,795.8( \pm 4,030.1)$ & $3,649.6( \pm 4,627.0)$ \\
\hline $\mathrm{TP}\left(\mathrm{ms}^{2}\right)$ & $7,024.2( \pm 6,344.5)$ & $4,989.0( \pm 5,902.0)$ \\
\hline \multicolumn{3}{|c|}{ Postintervention } \\
\hline $\mathrm{HF}\left(\mathrm{ms}^{2}\right)$ & I,07I.3 ( \pm I,277.2) & $774.5( \pm I, 23 \mid .5)$ \\
\hline $\operatorname{LF}\left(\mathrm{ms}^{2}\right)$ & $5,578.4( \pm 3,589.0)$ & $2,856.4( \pm 3,849.6)$ \\
\hline $\mathrm{TP}\left(\mathrm{ms}^{2}\right)$ & $7,269.1( \pm 5,231.7)$ & $4,136.0( \pm 4,893.3)$ \\
\hline \multicolumn{3}{|l|}{ Follow-up I } \\
\hline $\mathrm{HF}\left(\mathrm{ms}^{2}\right)$ & $868.6( \pm 734.2)$ & $871.0( \pm 1,894.9)$ \\
\hline $\operatorname{LF}\left(\mathrm{ms}^{2}\right)$ & $5,008.0( \pm 3,532.7)$ & $3,382.0( \pm 6,886.9)$ \\
\hline $\mathrm{TP}\left(\mathrm{ms}^{2}\right)$ & $6,476.6( \pm 4,6 \mid 1.0)$ & $4,981.0( \pm 9,385.2)$ \\
\hline \multicolumn{3}{|l|}{ Follow-up 2} \\
\hline $\mathrm{HF}\left(\mathrm{ms}^{2}\right)$ & $\mathrm{I}, 043.7( \pm \mathrm{I}, \mathrm{I} 20.3)$ & $\mathrm{I}, 067.6( \pm \mathrm{I}, 847.6)$ \\
\hline $\operatorname{LF}\left(m s^{2}\right)$ & $4,085.6( \pm 2,530.2)$ & $4,009.2( \pm 5,9 \mid 7.2)$ \\
\hline $\mathrm{TP}\left(\mathrm{ms}^{2}\right)$ & $6, I 10.1( \pm 4,023.6)$ & $5,657.6( \pm 8,009.5)$ \\
\hline
\end{tabular}

Notes: HRV was unchanged in both the control group and the biofeedback group. Data are expressed as mean \pm standard deviation. All HRV parameters were unchanged in both groups at each time point of assessment $(p=n s)$. In the control group, the term "postintervention" refers to the post-control period.

Abbreviations: HF, high frequency; HRV, heart rate variability; LF, low frequency; TP, total power; ns, not significant.

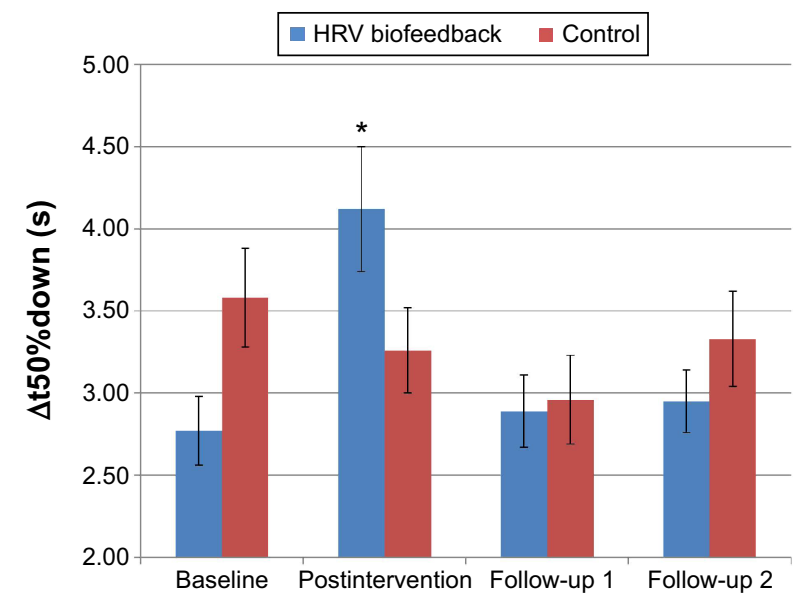

Figure 3 Vasomotor function: $\Delta \mathrm{t} 50 \%$ down.

Notes: After HRV biofeedback (but not in the control group), $\Delta \mathrm{t} 50 \%$ down was increased, indicating possible improved function of vasomotor autonomic nerve fibers. Data are presented as mean \pm standard deviation. $* P<0.05$ versus baseline. HRV biofeedback: $n=24$; control: $n=24$. In the control group, the term "postintervention" refers to the post-control period.

Abbreviations: $\Delta t 50 \%$ down, mean duration to $50 \%$ vasoconstriction; $H R V$, heart rate variability; ns, not significant.

cardiac autonomic function and psychiatric symptoms. ${ }^{10,11}$ In fact, an open-label study in 14 patients with depression undergoing HRV biofeedback training demonstrated an increase in HRV which was paralleled by a decrease of depressive symptoms and anxiety. ${ }^{11}$ This study was limited by lack of randomization and control group. However, its results are in line with our observation of decreased anxiety and tendentially increased HRV in patients with alcohol dependence. These beneficial effects on cardiac autonomic function and anxiety might also explain our observation of improved craving reduction following biofeedback, since craving was previously linked to increased anxiety

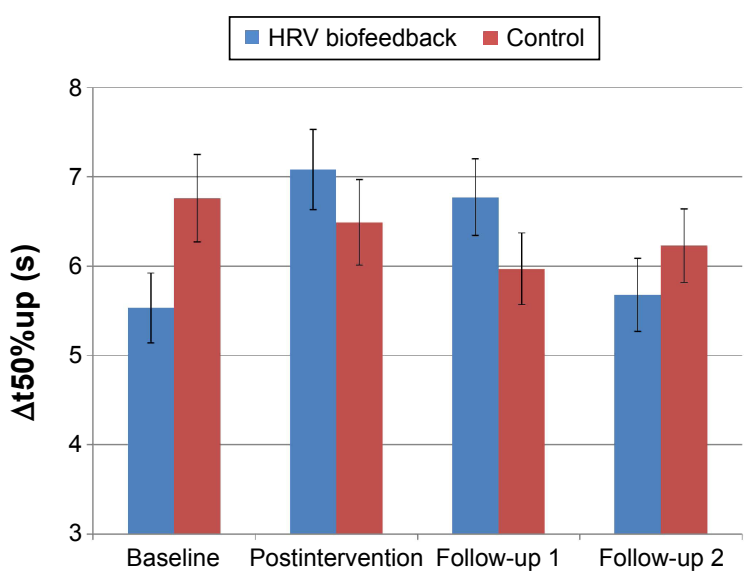

Figure 4 Vasomotor function: $\Delta \mathrm{t} 50 \%$ up.

Notes: $\Delta \mathrm{t} 50 \%$ up was unchanged in both the HRV biofeedback group and in the control group. Data are presented as mean \pm standard deviation. $P=n s$ compared to baseline and control. HRV biofeedback: $n=24$; control: $n=24$. In the control group, the term "postintervention" refers to the post-control period.

Abbreviations: $\Delta \mathrm{t} 50 \%$ up, duration to $50 \%$ redilation of cutaneous vessels; HRV, heart rate variability; ns, not significant. 
and impaired autonomic responsivity. ${ }^{22}$ Additionally, our observation of decreased craving after HRV biofeedback is consistent with previous studies on HRV biofeedback reporting reduced food craving in high food cravers and attenuated substance craving in individuals with posttraumatic stress disorders. ${ }^{23,24}$ These studies support the possible explanation of a reduction in craving through HRV biofeedback which is mediated by improvement of autonomic adaptive capacity. However, it remains to be answered how changes in the sympathetic/parasympathetic balance convert into alleviation of craving. Our observation of improved vasomotor function might indicate that a decrease in sympathetic outflow due to HRV biofeedback contributes to attenuation of craving, as the measures of cutaneous vasoconstriction used in our study constitute direct indicators of sympathetic vasomotor function. ${ }^{25,26}$ In accordance with this assumption, a previous controlled study showed increased sympathetic tone in patients with alcohol dependence when being exposed to craving induced by olfactory stimuli (sniffing of preferred alcoholic beverages). ${ }^{27} \mathrm{We}$ also noted an improvement in craving in the control group, which might be explained by the effectiveness of our standard rehabilitative treatment. However, the observation that improvement in craving occurred earlier in the HRV biofeedback group is encouraging, as it might indicate effectiveness of the intervention which even leads to an amplification of improvement of craving through standard care. Therefore, our data might support the potential use of HRV biofeedback as supplementary treatment of alcohol use disorder.

Even though the aforementioned studies lend support to a predominant sympathetic mechanism whereby HRV biofeedback improves craving, the anatomo-physiological link between decreased sympathetic tone and reduced craving remains to be elucidated. Peripheral sympathetic tone was shown to be regulated by sympathetic centers in the hypothalamus and brainstem, whereas the development of craving appears to be caused by functional alterations in limbic and prefrontal regions. Therefore, it might be speculated that communicating pathways between limbic/prefrontal and hypothalamic/brainstem regions might play a role in the beneficial effects of HRV biofeedback on craving. ${ }^{28,29}$

Although our observations of improved autonomic functions and decreased craving and anxiety appear to confirm the therapeutic value of HRV biofeedback in the treatment of patients with alcohol dependence, some limitations of our study have to be mentioned. First, the observed increase in HRV did not reach statistical significance. Since a type II error is unlikely, as our study population size was calculated prior to conduction of the study, we speculate that late-stage structural ethyl-toxic damage of cardiac autonomic fibers might have caused reduced responsivity to HRV biofeedback. However, even in our patients with late-stage alcohol use disorder, we observed a trend toward improved HRV. This beneficial effect might be stronger in earlier stages of the disease, where nerve fiber damage is less irreversible. Our results therefore warrant a follow-up study to investigate disease stage-specific effects of HRV biofeedback in patients with alcohol dependence. This follow-up investigation should also control for structural measures of autonomic fiber neuropathy, such as intraepidermal nerve fiber density assessment and explore the association of alcohol use disorder severity measures and observed effect sizes. Second, our study was limited by the lack of an active control condition using a sham intervention, where control patients are sitting in front of the computerized biofeedback system but are receiving neither breathing instructions nor actual HRV measurement. Although we refrained from an active control to avoid inpatients from the intervention group communicating breathing instructions to the control patients in our clinic, a sham intervention in the control group might have been more effective in controlling for potential placebo effects of the intervention. Therefore, follow-up research should include sham biofeedback and minimize the possibility of intergroup conversations on breathing instructions, eg, in a multicentric setting. The absence of an active control intervention in our control group might have jeopardized the robustness of physiological autonomic function measures and might explain the discrepancy of improved psychometric measures and only tendentially increased HRV. However, since our observations of decreased anxiety and craving indicate that HRV biofeedback might be effective as supplementary treatment in patients addicted to alcohol, this study may form a basis for a larger follow-up study which also includes an active control setting to elucidate to what extent these beneficial effects convert into improvement of autonomic functions. Third, since we performed a monocenter study in a limited number of patients, we cannot comment on the external validity of our findings, which should be assessed using a multicentric approach. Last, we used LDF to assess vasomotor function, a technique that is limited by interindividual variability and single-point assessment of cutaneous blood flow. Consequently, LDF was shown to be insensitive in the detection of vasomotor dysfunction in individual patients. However, the technique was also shown to reliably detect group differences between patients with neuropathic vasomotor dysfunction and healthy subjects. ${ }^{30-32}$ Consistent with these previous studies, we observed a group difference in vasomotor function assessment between patients undergoing HRV biofeedback 
and control patients. Future studies using HRV biofeedback should also include alternative vasomotor function techniques that have lower variability and allow for cutaneous blood flow evaluation with both temporal and spatial resolution, such as regression thresholding-based assessment of the laser Doppler imaging axon-reflex flare area. ${ }^{33}$

Taken together, our findings viewed in conjunction with the current literature suggest that HRV biofeedback might be an effective supplement to rehabilitative inpatient care of patients with alcohol addiction.

\section{Acknowledgments}

This study was part of a doctoral thesis. The authors thank Mrs Rita Polensky for her technical assistance in conducting the study. Additionally, the authors extend their sincere appreciation to Dr Günther Freier and Dr Ralph Deymann for their general support. Publication costs were funded by the Open Access Publication Funds of the Technische Universität Dresden.

\section{Disclosure}

The authors report no conflicts of interest in this work.

\section{References}

1. Wittchen HU, Jacobi F, Rehm J, et al. The size and burden of mental disorders and other disorders of the brain in Europe 2010. Eur Neuropsychopharmacol. 2011;21(9):655-679.

2. Johnson RH, Robinson BJ. Mortality in alcoholics with autonomic neuropathy. J Neurol Neurosurg Psychiatry. 1988;51(4):476-480.

3. Melgaard B, Somnier F. Cardiac neuropathy in chronic alcoholics. Clin Neurol Neurosurg. 1981;83(4):219-224.

4. Johnston LC, Patel S, Vankineni P, Kramer N. Deficient slowing of the heart among very heavy social drinkers. J Stud Alcohol. 1983;44(3): 505-514.

5. Low PA, Walsh JC, Huang CY, McLeod JG. The sympathetic nervous system in alcoholic neuropathy. A clinical and pathological study. Brain. 1975;98(3):357-364.

6. Quintana DS, Guastella AJ, McGregor IS, Hickie IB, Kemp AH. Heart rate variability predicts alcohol craving in alcohol dependent outpatients: further evidence for HRV as a psychophysiological marker of selfregulation. Drug Alcohol Depend. 2013;132(1-2):395-398.

7. Bottlender M, Soyka M. Impact of craving on alcohol relapse during, and 12 months following, outpatient treatment. Alcohol Alcohol. 2004;39(4):357-361.

8. Oslin DW, Cary M, Slaymaker V, Colleran C, Blow FC. Daily ratings measures of alcohol craving during an inpatient stay define subtypes of alcohol addiction that predict subsequent risk for resumption of drinking. Drug Alcohol Depend. 2009;103(3):131-136.

9. Schneekloth TD, Biernacka JM, Hall-Flavin DK, et al. Alcohol craving as a predictor of relapse. Am J Addict. 2012;21 Supp1 1:S20-S26.

10. Karavidas MK, Lehrer PM, Vaschillo E, et al. Preliminary results of an open label study of heart rate variability biofeedback for the treatment of major depression. Appl Psychophysiol Biofeedback. 2007;32(1): $19-30$.

11. Siepmann M, Aykac V, Unterdörfer J, Petrowski K, Mueck-Weymann M. A pilot study on the effects of heart rate variability biofeedback in patients with depression and in healthy subjects. Appl Psychophysiol Biofeedback. 2008;33(4):195-201.
12. Eddie D, Kim C, Lehrer P, Deneke E, Bates ME. A pilot study of brief heart rate variability biofeedback to reduce craving in young adult men receiving inpatient treatment for substance use disorders. Appl Psychophysiol Biofeedback. 2014;39(3-4):181-192.

13. Angelone A, Coulter NA Jr. Respiratory sinus arrhythmia: a frequency dependent phenomenon. J Appl Physiol. 1964;19:479-482.

14. Lehrer P, Carr RE, Smetankine A, et al. Respiratory sinus arrhythmia versus neck/trapezius EMG and incentive inspirometry biofeedback for asthma: a pilot study. Appl Psychophysiol Biofeedback. 1997; 22(2):95-109.

15. Anton RF, Moak DH, Latham P. The Obsessive Compulsive Drinking Scale: a self-rated instrument for the quantification of thoughts about alcohol and drinking behavior. Alcohol Clin Exp Res. 1995;19(1):92-99.

16. Nakovics H, Diehl A, Croissant B, Mann K. Modifications of the Obsessive Compulsive Drinking Scale (OCDS-G) for use in longitudinal studies. Addict Behav. 2008;33(10):1276-1281.

17. Derogatis LR, Cleary PA. Confirmation of the dimensional structure of the scl-90: a study in construct validation. J Clin Psychol. 1977;33(4): 981-989.

18. Beck A, Steer R, Ball R, Ranieri WJ. Comparison of Beck Depression Inventories -IA and -II in psychiatric outpatients. San Antonio: J Pers Assess. 1996;67(3):588-597.

19. Rechlin T, Claus D, Weis M. Heart rate analysis in 24 patients treated with $150 \mathrm{mg}$ amitriptyline per day. Psychopharmacology (Berl). 1994;116(1): 110-114.

20. Mück-Weymann M, Rechlin T. Reflexes of the cutaneous microcirculation in amitriptyline and in fluoxetine treated patients. Psychopharmacology (Berl). 1996;124(3):241-244.

21. Agelink MW, Malessa R, Weisser U, et al. Alcoholism, peripheral neuropathy (PNP) and cardiovascular autonomic neuropathy (CAN). J Neurol Sci. 1998;161(2):135-142.

22. McCusker CG, Brown K. The cue-responsivity phenomenon in dependent drinkers: 'personality' vulnerability and anxiety as intervening variables. Br J Addict. 1991;86(7):905-912.

23. Meule A, Freund R, Skirde A, Vögele C, Kübler A. Heart rate variability biofeedback reduces food cravings in high food cravers. Appl Psychophysiol Biofeedback. 2012;37(4):241-251.

24. Zucker TL, Samuelson KW, Muench F, Greenberg MA, Gevirtz RN. The effects of respiratory sinus arrhythmia biofeedback on heart rate variability and posttraumatic stress disorder symptoms: a pilot study. Appl Psychophysiol Biofeedback. 2009;34(2):135-143.

25. Johnson JM, Pérgola PE, Liao FK, Kellogg DL Jr, Crandall CG. Skin of the dorsal aspect of human hands and fingers possesses an active vasodilator system. J Appl Physiol (1985). 1995;78(3):948-954.

26. Hodges GJ, Johnson JM. Adrenergic control of the human cutaneous circulation. Appl Physiol Nutr Metab. 2009;34(5):829-839.

27. Kaplan RF, Cooney NL, BakerLH, GillespieRA, MeyerRE, Pomerleau OF. Reactivity to alcohol-related cues: physiological and subjective responses in alcoholics and nonproblem drinkers. J Stud Alcohol. 1985; 46(4):267-272.

28. Jansen AS, Nguyen XV, Karpitskiy V, Mettenleiter TC, Loewy AD. Central command neurons of the sympathetic nervous system: basis of the fight-or-flight response. Science. 1995;270(5236):644-646.

29. Schacht JP, Anton RF, Myrick H. Functional neuroimaging studies of alcohol cue reactivity: a quantitative meta-analysis and systematic review. Addict Biol. 2013;18(1):121-133.

30. Benarroch EE, Low PA. The acetylcholine-induced flare response in evaluation of small fiber dysfunction. Ann Neurol. 1991;29(6):590-595.

31. Low PA, Neumann C, Dyck PJ, Fealey RD, Tuck RR. Evaluation of skin vasomotor reflexes by using laser Doppler velocimetry. Mayo Clin Proc. 1983;58(9):583-592.

32. Freeman R. Autonomic peripheral neuropathy. Lancet. 2005;365(9466): 1259-1270.

33. Illigens BM, Siepmann T, Roofeh J, Gibbons CH. Laser Doppler Imaging in the detection of peripheral neuropathy. Auton Neurosci. 2013; 177(2):286-290. 


\section{Publish your work in this journal}

Neuropsychiatric Disease and Treatment is an international, peerreviewed journal of clinical therapeutics and pharmacology focusing on concise rapid reporting of clinical or pre-clinical studies on a range of neuropsychiatric and neurological disorders. This journal is indexed on PubMed Central, the 'PsycINFO' database and CAS, and is the official journal of The International Neuropsychiatric Association (INA). The manuscript management system is completely online and includes a very quick and fair peer-review system, which is all easy to use. Visit http://www.dovepress.com/testimonials.php to read real quotes from published authors.

\footnotetext{
Submit your manuscript here: http://www.dovepress.com/neuropsychiatric-disease-and-treatment-journal
} 